\title{
The spectral energy distribution of the Carina nebula from far infrared to radio wavelengths
}

\author{
M. Salatino, P. de Bernardis, S. Masi \\ Physics Department, Sapienza Università di Roma, p.le Aldo Moro 2, 00185 Roma, Italy \\ maria.salatino@roma1.infn.it \\ and \\ G. Polenta \\ ASI Science Data Center, ESRIN, via G. Galilei, 00044, Frascati, Italy \\ INAF-Osservatorio Astronomico di Roma, via di Frascati 33, I-00040 Monte Porzio Catone, \\ Italy
}

Received __; accepted _ 


\begin{abstract}
Multi-wavelength observations are mandatory to understand the physical properties of astrophysical sources. In this paper we use observations in the far infrared to radio range to derive the spectral energy distribution (SED) of the Carina nebula. To do this, we carefully subtract the irregularly varying diffuse emission from the Galactic plane, which can be of the order of $10 \%$ of the nebula flux at these wavelengths. We find that the far infrared SED can be modeled as emission from a dust population with a single temperature $T_{d}=\left(34.5_{-1.8}^{+2.0}\right)$, and with a spectral index of emissivity $\alpha=-1.37_{-0.08}^{+0.09}$. We also find a total infrared luminosity of the Nebula of $\left(7.4_{-1.4}^{+2.5}\right) \times 10^{6} L_{\odot}$ and, assuming a single temperature of the dust, a mass of the dust of $\left(9500_{-3500}^{+4600}\right) M_{\odot}$.
\end{abstract}

Subject headings: Infrared: ISM - Instrumentation: photometers - Methods: data analysis - Methods: statistical — radio continuum: ISM — Submillimeter: ISM 


\section{Introduction}

The Carina nebula (NGC 3372 or RCW 53) has one of the highest surface brightnesses of any nebula in the southern hemisphere. It contains the most luminous and one of the most massive stars of our Galaxy: eta Carinae, a LBV star. Its distance $(2.3 \mathrm{kpc})$ is known accurately, mainly through the expansion parallax (Allen \& Hillier 1993) of the Homunculus nebula (Smith 2005). The nebula has a particularly low extinction with respect to other star formation regions, and presents an anomalous reddening law (Smith 1987). The nebula hosts, moreover, more than 60 O stars, three WNL stars, an O2 supergiant, and a B1.5 supergiant which suggests the presence of a proto OB association (Smith 2006). The Carina nebula offers a unique laboratory to study different astrophysical problems: star formation, feedback on the star formation provided by young massive stars, as well as triggering of a second generation of star formation from the most massive ones; small-scale phenomena (evaporating protoplanetary disks, jets, cometary clouds, dust pillars, globules, Herbig-Haro jets (Smith et al. 2004), and photodissociation regions on the surfaces of molecular clouds (Rathborne et al. 2002)). High-quality data spanning from infrared (IR) to radio wavelengths show a heavily active star formation region in which the LBV star, as well as other massive ones, play a crucial role.

Knowledge of the nebula improves our knowledge of the interstellar medium, and, in particular, our models of interstellar dust. Observational cosmology has an interest in understanding dust properties too (Deschênes 2008; Dunklev et al. 2008; Lazarian \& Finkbeiner 2003; Masi et al. 2001): current measurements of the polarization of the cosmic microwave background (CMB) are limited by our limited ability to subtract foreground emission. Future experiments like the Planck and Herschel satellites (launched on 2009 May 14), and the PILOT (Bernard et al. 2007a.,b), EBEX (Oxley et al. 2004; Reichborn-Kjennerud et al. 2010), SPIDER (Montroy et al. 2006), and Balloon 
Observations of Millimetric Extragalactic Radiation and Geophysics (BOOMERanG) balloons, with their polarization surveys of the sky will provide data to understand dust properties: like the emissivities and the wavelength-dependence of the far infrared (FIR) polarized emission (Hildebrand et al. 1999; Lazarian \& Hoang 2007; Martin 2007). This knowledge is very much needed for a better extraction of the finest CMB polarization properties, which can provide information on cosmological inflation, in post-Planck missions like CMB-Pol (Baumann et al. 2009), B-Pol (de Bernardis et al. 2009) and COrE (http://www.core-mission.org).

The paper is organized as follows: in Section2, we briefly summarize the current knowledge of the Carina nebula. In Section 3 we present the data we used for our analysis. The subtraction of the background is shown to be, with the calibration error, the dominant source of error for the measured fluxes (Section 4). In Section[5, we present the FIR to radio spectral energy distribution (SED), and the best fit we performed using $\chi^{2}$ minimization and a Monte Carlo Markov Chain Metropolis-Hastings (MCMCMH) algorithm for the estimate of the confidence intervals. We derive some global properties of the nebula and compare our results to previous literature data in Section 6, and we conclude summarizing the results and future activities in Section:7.

\section{The SED and the global properties}

Smith \& Brooks (2007) have integrated IRAS (FIR) data and the Parkes (radio) data to estimate the flux of the Carina nebula in a box $1^{\circ} 58^{\prime} \times 2^{\circ} 45^{\prime}$ wide. The resulting SED is described by three graybody components having temperatures of 220 , 80 , and $35 \mathrm{~K}$ with emissivity $\propto \lambda^{-1}$, and by an optically thin thermal bremsstrahlung radio continuum emission component $\left(\propto \nu^{-0.1}\right)$. Their analysis used the two data at 60 and $100 \mu \mathrm{m}$ to fit the Carina nebula SED in the wavelength range (20-5000) $\mu \mathrm{m}$, with two free parameters (temperature 
and emissivity), assuming a spectral index of emissivity -1; the single measurement at radio wavelengths is used to measure the amplitude of the thermal bremsstrahlung component.

Luminosity at these wavelengths is independent of dust grains radius and emissivity as long as the radius of the grains is small, $<0.2 \mu \mathrm{m}$ (Draine \& Lee 1984). With a typical grain density, $\rho$, of about $3 \mathrm{~g} \mathrm{~cm}^{-3}$ and a temperature $T$, the dust mass which emits an IR luminosity, $L_{I R}$, is (Smith \& Gehrz 2005):

$$
M_{d u s t}\left(M_{\odot}\right)=\frac{122 \rho}{3 \sigma_{S B} T^{6}} L_{I R}\left(L_{\odot}\right)
$$

with $\sigma_{S B}$ being the Stefan-Boltzmann constant expressed in cgs units. For the dust population at $35 \mathrm{~K}$ Smith \& Brooks (2007) found a luminosity and a mass equal to $7.6 \times 10^{6} L_{\odot}$ and $9600 M_{\odot}$, respectively. In the following we keep the same area considered by Smith \& Brooks (2007) and we estimate the integrated flux of additional experiments carried out at different wavelengths.

Studies of the Carina nebula at wavelengths shorter than $60 \mu \mathrm{m}$ are already present in literature (Cox et al. 1995; Morris et al. 1999; Rathborne et al. 2002; Smith \& Brooks 2007). In this work we demonstrate that in the FIR portion of the spectrum the B98 and Wilkinson Microwave Anisotropy Probe (WMAP) data improve our knowledge of the nebula.

\section{Multiwavelength observations}

In this section, we describe the multiwavelength data we used and how they have been analyzed. Although we used different experiments, some parts in the data analysis are the same.

For each experiment the average value of the background has been estimated as described in Section 4. For the background estimation, we have also considered a possible 
contribution from CMB anisotropy, but we found that it is negligible, given the large number of independent sky regions present in our integration box.

\section{1. $\mathrm{COBE}$}

The DIRBE instrument of the COBE satellite has produced infrared absolute sky brightness maps of the Carina nebula in the wavelength range 1.25 to $240 \mu \mathrm{m}$ with an instantaneous field of view of $0 .^{\circ} 7 \times 0 .^{\circ} 7$ (Hauser et al. 1998a). For our analysis we used data at wavelengths longer than $60 \mu \mathrm{m}$. The quadrilateralized spherical cube projection of the DIRBE maps has been converted into the Hierarchical Equal Area isoLatitude PIXelization, HEALPIX (Gorski et al. 2005); this has required a change of pixel size with respect to the original: from about $0 .^{\circ} 32 \mathrm{o}$ to $27 . ' 5$ (nside=128). From these data we have calculated the maps and the integrated fluxes in the same box considered by Smith \& Brooks (2007). The results are shown in Figure1 and Table4. The errors in the calculated fluxes are the quadrature sum of the uncertainty in the absolute gain calibration, the uncertainty in the instrumental offset (zero point), the standard deviation of the weighted average photometry values (Hauser et al. 1998b), and the contribution from both the background diffuse emission of the Galaxy (see Section 4) and the rms CMB anisotropy.

\subsection{IRAS}

IRAS has observed, at wavelengths of 60 and $100 \mu \mathrm{m}$, the Carina nebula with an angular resolution of $1 .^{\prime} 5 \times 4 .^{\prime} 7$, and $3 .^{\prime} 0 \times 5 .^{\prime} 0$, respectively (Neugebauer et al. 1984$)$. We

have used the latest processed IRAS images IRIS (Deschênes \& Lagache 2005): they have an absolute calibration coherent with DIRBE, a better zodiacal light subtraction, and better destriping with respect to the original ISSA maps (Wheelock et al. 1993). A preliminary 
comparison with the original ISSA data gave flux values $22 \%$ and $7.9 \%$ smaller than the IRIS data at 60 and $100 \mu \mathrm{m}$, respectively, the discrepancy with the IRIS data is $1.5(0.3) \sigma$ at $60(100) \mu \mathrm{m}$. For coherence with the other experiments, that are in the HEALPIX pixelization, we used the IRIS data. The resolution is 1.'7 (nside 2048) (Gorski et al. 2005). The brightness map in our integration box is reported in Figure 1 and the resulting flux in Table4,

\subsection{BOOMERanG 98}

The stratospheric balloon experiment BOOMERanG 98, hereafter B98, (Crill et al. 2003 ) observed the Carina nebula at frequencies of 90, 150, 220, and $410 \mathrm{GHz}$ (Coble et al. 2005) with an angular resolution of about 18.'1, 11.'7, 15.'9 and 15.'0, respectively. For the $410 \mathrm{GHz}$ channel the calibration error is $25 \%$ (Masi et al. 2001), for the others $10 \%$

(Ruhl et al. 2003). Since the B98 survey does not fill completely the Smith integration box, the missing region (white region in B98 maps, Figure1) is filled with the average signal from an observed background region of area $0 .{ }^{\circ} 2 \times 0 .{ }^{\circ} 2$ (see Tab.11). The missing area is at most $10 \%$ of the integration box, in a low background region. From the WMAP7 map at a similar frequency $(94 \mathrm{GHz})$ we see that neglecting completely the contribution from this area the flux decreases by $1.2 \%$, so the error we introduce with our procedure is certainly less than $1 \%$ and in any case is smaller than the errors introduced by the background subtraction procedure (see Section44). Fluxes have been corrected for the instrumental response of the telescope using the transmissions functions of the 16 B98 filters. The signal, in Jansky, for each channel $i, S_{i}$, is given by

$$
S_{i}=\frac{k_{A, i} T_{C M B}}{\int_{\Delta \nu_{i}} e_{i}(\nu) B(\nu) \frac{x e^{x}}{e^{x}-1} \Delta \Omega d \nu} \int_{\Delta \nu_{i}} e_{i}(\nu) I_{i}(\nu) d \nu,
$$

in which $T_{C M B}=2.73 \mathrm{~K}$ is the temperature of the CMB (Fixsen et al. 1996),$x=h \nu /(k T)$ (with $k$ being the Boltzmann constant and $h$ being the Planck constant), $k_{A, i}$ is the 
calibration factor of the $i$ th channel, $e_{i}(\nu)$ the filter transmission function of the $i$ th channel, and $I_{i}(\nu)$ is the theoretical emission model.

Table 1: Regions Observed by B98 in Different Frequency Channels, and Used to Fill Missing Regions in the B98 Survey; the Columns List Respectively the B98 Channels, and the Galactic Coordinates of the Edges of the Regions: $\ell_{\min }, \ell_{\max }, b_{\min }, b_{\max }$.

\begin{tabular}{ccccc}
\hline \hline Channel & $\ell_{\min }$ & $\ell_{\max }$ & $b_{\min }$ & $b_{\max }$ \\
\hline B90A, B90B & $287^{\circ} .9$ & $288^{\circ} .1$ & $-1^{\circ} .8$ & $-1^{\circ} .6$ \\
B150A, B150B, B150A2, B150B1, B150B2 & $288^{\circ} .3$ & $288^{\circ} .5$ & $-2^{\circ} .1$ & $-1^{\circ} .9$ \\
B150A1 & $287^{\circ} .6$ & $287^{\circ} .8$ & $-2^{\circ} .1$ & $-1^{\circ} .9$ \\
B220A1, B220B1, B220B2 & $287^{\circ} .9$ & $288^{\circ} .1$ & $-1^{\circ} .8$ & $-1^{\circ} .6$ \\
B220A2 & $288^{\circ} .3$ & $288^{\circ} .5$ & $-2^{\circ} .1$ & $-1^{\circ} .9$ \\
B410A2, B410B1 & $288^{\circ} .3$ & $288^{\circ} .5$ & $-2^{\circ} .1$ & $-1^{\circ} .9$ \\
B410A1 & $288^{\circ} .3$ & $288^{\circ} .5$ & $-1^{\circ} .8$ & $-1^{\circ} .6$ \\
\hline
\end{tabular}

We have assumed a typical power law, $I_{i}(\nu)=I_{0, i}\left(\nu / \nu_{0, i}\right)^{\beta}$, where $I_{0, i t h}$ is the flux measured at frequency $\nu_{0, i}$, the central frequency of the $i$ th channel. The integration is performed over the entire band of the filter, $\Delta \nu_{i}$. The color corrections (Table2) are less than $1 \%$ for an optically thin thermal bremsstrahlung $(\beta=-0.1)$, Rayleigh-Jeans spectrum $(\beta=2)$ or a power law with $\beta=3$. Since in the B98 wavelength range (excluding the $90 \mathrm{GHz}$ channels) we expect thermal dust emission to be predominant over bremsstrahlung emission, we carried our analysis with the color corrections relative to $\beta=2$. B98 was a scanning instrument. To remove instrumental offsets and drifts, we have high-pass filtered the time-ordered data. The cutoff of the high-pass filter corresponds to an angle $\theta_{C}$ in the sky, so the B98 maps we have used do not contain structures at scales larger than $\theta_{C}$. We have verified that the fluxes in our target region are not significantly affected by filtering if $\theta_{C} \gtrsim 10^{\circ}$ (see Table2). 
Table 2: Variations of the Integrated Fluxes (Color-corrected) Measured at $150 \mathrm{GHz}$ for Different Spectral Index of SED, $\beta$, and Different Cutoff $\theta_{C}$ of the High-pass Filter Applied to the Time-ordered Data. The fluxes are corrected for the background emission $(n=5$, see Section 4 (1).

\begin{tabular}{ccc}
\hline \hline Filter & $\beta$ & Flux (Jy) \\
\hline $5^{\circ} .0$ & -0.1 & $920 \pm 150$ \\
& 2.0 & $920 \pm 150$ \\
& 3.0 & $910 \pm 150$ \\
$10^{\circ} .0$ & -0.1 & $1630 \pm 190$ \\
& 2.0 & $1620 \pm 190$ \\
& 3.0 & $1610 \pm 190$ \\
$15^{\circ} .0$ & -0.1 & $1920 \pm 210$ \\
& 2.0 & $1920 \pm 210$ \\
& 3.0 & $1900 \pm 210$ \\
\hline
\end{tabular}


The errors in the fluxes are the quadrature sum of the standard deviations of the measured signals, the calibration uncertainties, and the Galactic background contributions. Depending on the channel, the calibration error dominates on the background one and vice versa (Table 4) while the statistical error represents a small contribution to the total.

The final integrated fluxes are computed as averages of different channels with the same frequency: one for $90 \mathrm{GHz}$, four for $150 \mathrm{GHz}$, three for $220 \mathrm{GHz}$, and three for $410 \mathrm{GHz}$. The results are reported in Table 4 and Figure1.

\subsection{WMAP}

The Carina nebula has been observed by the WMAP satellite (Bennett et al. 2003) at the frequencies: $23 \mathrm{GHz}$ ( $K$-band), $33 \mathrm{GHz}$ ( $K a$-band), $41 \mathrm{GHz}$ ( $Q$-band), $61 \mathrm{GHz}$ ( $V$-band) and $94 \mathrm{GHz}$ ( $W$-band); the seven-year release data, hereafter WMAP7, provide maps of the nebula with FWHM angular resolution of about $0^{\circ} .81,0^{\circ} .62,0^{\circ} .48,0 .^{\circ} 33$, and $0 .{ }^{\circ} 21$,

respectively (Hinshaw et al. 2007, 2009). We used the temperature (Stokes I parameter) maps per frequency band obtained from the Lambda archive (http://lambda.gsfc.nasa.gov/). The fluxes have been corrected for the instrumental responses of each of the 20 differential radiometers; we averaged the bandpass frequency responses of all the radiometers in each frequency band. The errors have been estimated as the quadrature sum of statistical error, background uncertainty, calibration error (0.5\%) and pixel noise (Hinshaw et al. 2009). The results for the integrated fluxes are reported in Table 4 and the maps in Figure1.

\subsection{Spitzer}

The MIPS instrument on board of the Spitzer satellite (Spitzer archive: http://archive.spitzer.caltech.edu/) has observed the Carina nebula at far infrared 
wavelengths of 24,70, and $160 \mu \mathrm{m}$ (Rieke et al. 2004). However, the photoconductors of MIPS were significantly saturated by the strong emission of the nebula, so we decided to exclude these data from our analysis.

\subsection{Multiwavelength maps}

The maps resulting from our analysis of all the experiments are displayed in Figure1; they are labeled with their HEALPIX pixel scale. The COBE maps, nside=128, have been filtered with a Gaussian low-pass filter $\left(\mathrm{FWHM}=0^{\circ} .35\right)$. Because of the low resolution of the instruments, the inner morphology of the Carina nebula is not resolved in the COBE-DIRBE maps and in the three lower frequency channels of WMAP. The lower resolution of the B98 instrument, with respect to the experiments analyzed by Smith \& Brooks (2007), does not allow a real pixel to pixel comparison: there is, nevertheless, significant morphological resemblance between the IRAS-IRIS, the B98 maps and the 61 and $94 \mathrm{GHz}$ channels of WMAP7.

\section{Background subtraction}

The region around the Carina nebula, near the Sagittarius-Carina spiral arm, is rich in massive star formation and HII regions: NGC 3603, NGC 3576, NGC 3572, NGC 3324, NGC 3293 and RCW 49 (Smith \& Brooks 2008). With respect to these regions Carina is larger, brighter, closer (2.3 kpc against 6-8 kpc) and has a lower extinction and reddening; therefore, its emission should dominate.

Background contributions (CMB, sky and irregularly varying diffuse emission) have been studied averaging flux values with two different numbers of background regions (labeled with the index $n$ ). In both cases we use six sets of reference regions, each set 
including five regions $(n=5)$ and two regions $(n=2)$. All regions have an area of $0 .^{\circ} 5 \times 0 .^{\circ} 5$ (see Table 3). For a given $n$, the reference regions are labeled with the index $m$. For $n=5$ the regions have been randomly chosen around the Carina nebula, for $n=2$ the regions are close to the nebula and symmetrically located with respect to its center. Only regions observed by all the experiments and close to the Carina nebula have been selected. We computed the average of the fluxes of all the regions in each set. We used the average of all the sets as an estimate of the background, and its standard deviation as an estimate of the error on the background.

Table 3: Selected Sky Areas for the Study of the Diffuse Background Contribution. Top part: $n=5$, bottom part: $n=2 ; m$ is the index of the region, $\ell_{i}, b_{i}(i=1,5)$ are the galactic coordinates (in degrees) for the centers of the regions.

\begin{tabular}{|c|c|c|c|c|c|c|c|c|c|c|}
\hline$m$ & $\ell_{1}$ & $b_{1}$ & $\ell_{2}$ & $b_{2}$ & $\ell_{3}$ & $b_{3}$ & $\ell_{4}$ & $b_{4}$ & $\ell_{5}$ & $b_{5}$ \\
\hline 1 & 289.75 & 1.25 & 285.15 & -3.75 & 285.15 & 1.25 & 287.20 & 1.25 & 285.75 & -2.35 \\
\hline 2 & 289.75 & 2.25 & 286.15 & -3.75 & 285.15 & 2.25 & 287.20 & 2.25 & 285.75 & -3.35 \\
\hline 3 & 289.75 & 3.25 & 284.75 & -2.25 & 288.25 & 1.25 & 284.25 & -3.75 & 285.55 & 3.25 \\
\hline 4 & 288.75 & 1.75 & 286.25 & 1.75 & 284.25 & -4.25 & 285.25 & -2.25 & 287.75 & 3.75 \\
\hline 5 & 288.25 & 3.25 & 284.25 & -2.75 & 284.25 & 1.75 & 289.75 & 4.75 & 289.25 & 1.75 \\
\hline \multirow[t]{8}{*}{6} & 286.25 & 4.75 & 284.75 & -3.25 & 284.25 & 1.25 & 290.25 & 2.75 & 284.75 & 2.95 \\
\hline & & & $m$ & $\ell_{1}$ & $b_{1}$ & $\ell_{2}$ & $b_{2}$ & & & \\
\hline & & & 1 & 285.25 & -3.75 & 289.75 & 2.25 & & & \\
\hline & & & 2 & 286.25 & -2.25 & 288.75 & 0.75 & & & \\
\hline & & & 3 & 284.75 & -3.25 & 290.75 & 1.75 & & & \\
\hline & & & 4 & 284.25 & -3.75 & 290.75 & 2.25 & & & \\
\hline & & & 5 & 285.75 & -3.25 & 289.25 & 1.75 & & & \\
\hline & & & 6 & 285.25 & -2.75 & 289.75 & 1.25 & & & \\
\hline
\end{tabular}


An indicator of the effect of background fluctuations is the ratio between the standard deviation of the average background and the nebula flux (corrected for the background). When computed for all the regions of Table 3 this indicator has a trend increasing with wavelength, and is maximum between the $240 \mu \mathrm{m}$ DIRBE and the $410 \mathrm{GHz}$ B98 images; on the contrary this indicator is basically independent of the considered sky region (Figure2).

The background contribution is more dependent on the number of regions considered for the analysis; this seems to be true for all the experiments. Similar background trends are found considering a small number of sky regions (maximum three) with different solid angles $\left(\right.$ from $1^{\circ} \times 1^{\circ}$ up to $\left.6^{\circ} \times 2^{\circ}\right)$.

In both cases, the WMAP data are the least affected by the background, followed by B98, IRAS and COBE. The background disagreement between the B98 $90 \mathrm{GHz}$ and WMAP5 $94 \mathrm{GHz}$ is due to the high-pass filtering of the BOOMERanG data.

Our analysis seems to indicate, for the irregularly varying emission surrounding the Carina nebula, a significative spectral dependence and a large scale nature (larger than $\left.6^{\circ} \times 4^{\circ}\right)$

The error on the background in case $n=5$ is about 1.5 times smaller than in case $\mathrm{n}=2$. Since case $n=5$ has better statistical significance, we have selected this configuration for the subtraction of the background in our integrated flux analysis. However, we have verified that all the results we give below remain very similar if we use the background estimates of case $n=2$.

\section{SED of the Carina Nebula, from FIR to cm-wavelengths}

The integrated fluxes, with the background subtraction described in Section 4, are reported in Table4. In Figure 4 we plot the SED of the Carina nebula. At 60 and $100 \mu \mathrm{m}$, 
Table 4: Integrated Fluxes for $n=5$. For a given wavelength of observation, $\lambda$, of an experiment, we report, in the order: experiment, flux, total, statistical, background, and calibration

\begin{tabular}{|c|c|c|c|c|c|c|}
\hline$\lambda$ & Experiment & Flux (Jy) & Error $(\%)$ & Stat $(\%)$ & $\operatorname{Bckg}(\%)$ & Cal $(\%)$ \\
\hline $60 \mu \mathrm{m}$ & IRAS (IRIS) & $(7.5 \pm 0.8) \times 10^{5}$ & 10.4 & 0.04 & 1.0 & 10 \\
\hline $60 \mu \mathrm{m}$ & COBE & $(5.7 \pm 0.6) \times 10^{5}$ & 10.5 & 0.2 & 1.4 & 10 \\
\hline $100 \mu \mathrm{m}$ & IRAS (IRIS) & $(9.7 \pm 1.3) \times 10^{5}$ & 13.7 & 0.03 & 2.2 & 13 \\
\hline $100 \mu \mathrm{m}$ & COBE & $(8.0 \pm 1.1) \times 10^{5}$ & 13.8 & 0.2 & 2.6 & 13 \\
\hline $140 \mu \mathrm{m}$ & COBE & $(9.0 \pm 1.0) \times 10^{5}$ & 11.4 & 0.1 & 4.1 & 11 \\
\hline $240 \mu \mathrm{m}$ & COBE & $(4.1 \pm 0.5) \times 10^{5}$ & 13.1 & 0.1 & 6.1 & 12 \\
\hline $731 \mu \mathrm{m}$ & B98 & $(1.6 \pm 0.4) \times 10^{4}$ & 26.6 & 0.2 & 9.2 & 25 \\
\hline $1.4 \mathrm{~mm}$ & B98 & $(4.2 \pm 0.5) \times 10^{3}$ & 12.8 & 0.3 & 8.0 & 10 \\
\hline $2.0 \mathrm{~mm}$ & B98 & $(1.6 \pm 0.2) \times 10^{3}$ & 11.8 & 0.6 & 6.3 & 10 \\
\hline $3.2 \mathrm{~mm}$ & WMAP7 & $(10.2 \pm 0.2) \times 10^{2}$ & 2.4 & $1.1 \cdot 10^{-3}$ & 2.3 & 1.7 \\
\hline $3.3 \mathrm{~mm}$ & B98 & $(0.9 \pm 0.1) \times 10^{3}$ & 11.8 & 0.5 & 6.2 & 10 \\
\hline $5.0 \mathrm{~mm}$ & WMAP7 & $(9.5 \pm 0.2) \times 10^{2}$ & 1.9 & $9.7 \cdot 10^{-4}$ & 1.8 & 1.6 \\
\hline $7.3 \mathrm{~mm}$ & WMAP7 & $(9.6 \pm 0.2) \times 10^{2}$ & 1.9 & $5.1 \cdot 10^{-4}$ & 1.8 & 1.6 \\
\hline $9.7 \mathrm{~mm}$ & WMAP7 & $(9.9 \pm 0.2) \times 10^{2}$ & 1.9 & $5.4 \cdot 10^{-4}$ & 1.9 & 1.6 \\
\hline $1.3 \mathrm{~cm}$ & WMAP7 & $(9.7 \pm 0.2) \times 10^{2}$ & 2.0 & $2.6 \cdot 10^{-4}$ & 2.1 & 1.6 \\
\hline
\end{tabular}


the fluxes from $C O B E$ and from the IRAS-IRIS are mutually consistent within 2 and $1 \sigma$, respectively. The IRAS-IRIS flux at $60 \mu \mathrm{m}$ is $1 \sigma$ away from Smith \& Brooks (2007), and the COBE one is at $2.9 \sigma$. The IRAS-IRIS and the COBE fluxes at $100 \mu \mathrm{m}$ are at 2.4 and $3.5 \sigma$, respectively, with respect to Smith \& Brooks (2007). The extrapolation at $3.4 \mathrm{~cm}$ from B98 $90 \mathrm{GHz}$ and all WMAP7 data is about $10^{3} \mathrm{Jy}$, at $1.6 \sigma$ from the Parkes data at the same wavelength (Smith \& Brooks 2007). This could be due to either an absolute calibration / offset problem in the Parkes data, or the presence of a further background rapidly decreasing with frequency.

We fitted the SED, excluding the COBE points at 60 and $100 \mu \mathrm{m}$, using the sum of a graybody (with an emissivity power law $\left(\lambda / \lambda_{1}\right)^{\alpha_{1}}$ and amplitude $\left.e_{1}\right)$ and an optically thin bremsstrahlung component:

$$
F_{\nu}=e_{1} \frac{2 h \nu^{3}}{c^{2}} \frac{1}{\left(e^{h \nu / k T_{1}}-1\right)}\left(\frac{\lambda}{\lambda_{1}}\right)^{\alpha_{1}} \Omega_{b}+a_{s}\left(\frac{\nu}{\nu_{0}}\right)^{-0.1},
$$

where $F_{\nu}$ is the flux density, in $\mathrm{Jy}$, and $\Omega_{b}$ the area, in sr, of the integration box. A chi-square minimization with four free parameters $\left(\chi^{2} ; 4\right)$ (the emissivity amplitude at $\lambda_{1}=100 \mu \mathrm{m}, e_{1}$; the emissivity spectral index $\alpha_{1}$; the temperature of the graybody component $T_{1}$, and the amplitude of the free-free component $a_{s}$ at the Parkes frequency $\left.\nu_{\mathbf{0}}\right)$ gives: $\chi_{\text {reduced }\left(\chi^{2} ; 4\right)}^{2}=1.16, e_{1\left(\chi^{2} ; 4\right)}=(1.09 \pm 0.13) \times 10^{-3}, T_{1\left(\chi^{2} ; 4\right)}=(35.0 \pm 2.0) \mathrm{K}$, $\alpha_{1\left(\chi^{2} ; 4\right)}=-1.32 \pm 0.09$, and $a_{s\left(\chi^{2} ; 4\right)}=(1100 \pm 21) \mathrm{Jy}$. The bremsstrahlung amplitude is well constrained; the IRAS/COBE data bind the peak, while the B98 ones constrain the long wavelength trend of the graybody law.

We studied the correlation between parameters using an MCMCMH algorithm (Andrieu et al. 2003; Lewis \& Bridle 2002; MacKay et al. 2002). The resulting contour plots, showing Confidence Levels (CL) regions of $68.3 \%$, 95.4, and 99.7\% are reported in Figure3,

Our parameters are well determined even without the introduction of priors in the 
algorithm. The graybody parameters are, as expected, not correlated to the bremsstrahlung one; the temperature, the emissivity amplitude and its spectral index, instead, are partially correlated. The estimates for the parameters $(m c m c ; 4)$, calculated as the average value of the random numbers accepted by the algorithm, are: $e_{1(m c m c ; 4)}=\left(1.10_{-0.28}^{+0.38}\right) \times 10^{-3}$, $T_{1(m c m c ; 4)}=\left(34.5_{-1.8}^{+2.0}\right) \mathrm{K}, \alpha_{1(m c m c ; 4)}=\left(-1.37_{-0.08}^{+0.09}\right)$, and $a_{s_{1}(m c m c ; 4)}=\left(1113_{-15}^{+12}\right)$ Jy with a reduced $\chi_{\text {reduced(mcmc; })}^{2}=1.009$. As expected the MCMCMH provides a better chi-square since the algorithm is not limited by the resolution of the parameters grid step as in a simple grid-based chi-square minimization.

We also investigated the possibility that the SED of the Carina nebula could be described by two graybodies: we added the free parameters $e_{2}, T_{2}$, and $\alpha_{2}$, normalizing the second emissivity power law at $\lambda_{2}=2000 \mu \mathrm{m}$. The minimization of the $\chi^{2}$-square fit provides hints for the possible existence of a colder dust component, with temperature of about $18 \mathrm{~K}$ and emissivity of about $2 \times 10^{-5}$, while the warmer component would have a temperature of about $40 \mathrm{~K}$, with a lower emissivity with respect to the case of a single population.

However, the results are unstable because of the large number of free parameters with respect to the number of independent data and the intrinsic degeneracy between the temperature and the spectral index (Dupac et al. 2003); moreover, the improvement of the fit with respect to the four parameters case is not significant.

We used these results as input for an MCMCMH run with the same seven free parameters. We restricted the parameters space, adding uniform priors for the conditional probabilities; therefore we set $T_{2}>0, e_{2}>0$ and $\alpha_{2}$ varying in the range $[-2.4 ;-0.8]$. The latter range has been selected following the temperature dependence of the submillimeter spectral index derived by PRONAOS data (Dupac et al. 2003).

The graybody parameters still remain not correlated to $a_{s}$, but the small number of 
experimental data, with respect to the free parameters, is not sufficient to constrain the parameters of the second graybody and to study their correlation properties. As a check of the instability of this result, we performed $100 \mathrm{MCMCMH}$ runs, with the same nstep and burn_in, and compare the results of the first run to the other 99 ones. Large variations were found, for all but the $a_{s}$ parameter, and, in any case, the lowest $\chi^{2}$ value we found was larger than the previous one.

We tried to eliminate the parameter $\alpha_{2}$ using its dependence from $T_{2}$ (see, e.g., Paradis et al. (2010); Veneziani et al. (2010)); following Dupac et al. (2003) we tried

$$
\alpha_{2}=-\frac{1}{0.4+0.008 T_{2}} \text {. }
$$

An MCMCMH with six parameters confirms the results of the MCMCMH with seven parameters of a warmer dust component with a lower emissivity and a second colder dust population with temperature of about $18 \mathrm{~K}$ and emissivity of about $2 \times 10^{-5}$. This is not a significant improvement over the four parameters fit, given the reduction of the degrees of freedom.

We conclude that the data, while not excluding it, do not require an additional cold dust component.

We also checked how the background removal method affects the best fit parameters. In case $n=2$ we find a reduced $\chi^{2}$ lower by $10 \%$ with respect to case $n=5$, and a temperature $T_{1}$ warmer by $2 \mathrm{~K}$. All the best fit parameters are consistent with ones obtained in case $n=5$ within $1 \sigma$.

Integrating the spectral flux density describing the $34.5 \mathrm{~K}$ dust population and considering an average distance of the nebula equal to $2.3 \mathrm{kpc}$ (Allen \& Hillier 1993), we calculate the total IR luminosity for this dust population and the corresponding dust mass (Section 2) obtaining $\left(7.4_{-1.4}^{+2.5}\right) \times 10^{6} L_{\odot}$ and $\left(9500_{-3500}^{+4600}\right) M_{\odot}$; both are well consistent with 
the corresponding estimates of Smith \& Brooks (2007). In presence of two dust grains populations the total luminosity is consistent, within $1 \sigma$, with the Smith \& Brooks (2007) estimate. On the contrary, the mass determination is strongly dependent on the assumption of a single dust population. Two dust populations hypothesis does not affect the luminosity because the SED remains very similar, but has a significant impact on the mass estimation as the mass strongly depends on the temperature. The nebula mass estimate increases up to $9.2 \times 10^{5}$ solar masses if we add a second population with temperature of about $18 \mathrm{~K}$ (see above).

\section{Discussion and future perspectives}

The determination of the SED improves our knowledge of this star forming region. In fact, the spectral index is a signature of a given type of dust (amorphous carbon and/or porous aggregates of silicates and graphite rather than silicates in ice mantles), and the parameters describing the SED are used to estimate the luminosity and the mass of a given dust population.

Compared to Smith \& Brooks (2007), our results agree with the presence of a dust population with temperature of $35 \mathrm{~K}$, although the fits with a double dust population might suggest the presence of slightly warmer dust (about $40 \mathrm{~K}$ instead of $35 \mathrm{~K}$ ). This results in a significantly lower estimate of the mass for the warm dust component. At variance with Smith \& Brooks (2007), in our analysis the emissivity spectral index is not assumed a priori, but is obtained from the data. A spectral index around -1.4 was predicted (Koike et al. 1980) from laboratory measurements on amorphous carbon, although this can be produced also by silicates, amorphous carbon and graphite bounded together in a porous aggregate (Mathis \& Whiffen 1989). A spectral index around -1 was also measured at $1.3 \mathrm{~mm}$ for observations towards two pre-main-sequence stars in the Taurus dark clouds; there thermal 
emission is due to small particles embedded in disks of gas and dust surrounding the stars (Beckwith et al. 1990). Values between $(-1-0)$ and $(-2--1)$ are found, respectively, in submillimeter and millimeter wavelength observations toward T Tauri and FU Orionis stars (Weintraub et al. 1989); the former range is related to extended dust clouds, the latter range is typical of dust in molecular clouds and compact HII regions. PRONAOS observations of Orion, M17, $\rho$ Ophiuchi and Cygnus B show a spectral index around -1 (Dupac et al. 2003), again consistent with our results. Early Planck results for diffuse dust in our Galaxy (Planck Collaboration 2011a) show a spectral index (-1.8), steeper than our value. However, the dust population sampled here is in a different (warmer) environment. In fact, indications of a steepening of the spectral index for colder temperatures, like in Equation (4), have been noted by several analysis (see e.g. Paradis et al. (2010); Veneziani et al. $(2010))$.

For our 4 parameters fits, our best fit spectral indexes are: $\alpha_{1\left(\chi^{2} ; 4\right)} \simeq-1.32$ and $\alpha_{1(m c m c ; 4)} \simeq-1.37$. Using the temperatures estimated from the fit, Equation (4), instead, would predict $-1.47(1.6 \sigma$ away from ours) and $-1.48(1.1 \sigma)$, in general in reasonable agreement with the data.

From a simple equilibrium equation we can estimate the temperature of the grains, $T_{g}$, as:

$$
T_{g} \sim \sqrt[4]{\frac{<Q_{U V}>}{<Q_{I R}>} \frac{L_{s}}{16 \pi \sigma_{S B} d^{2}}} \sim 40 \mathrm{~K}
$$

with $<Q_{U V(I R)}>$ the blackbody-averaged Q-factor in the UV (IR) wavelengths (Draine 1981; Draine \& Lee 1984), $L_{s} \sim 2 \times 10^{7}$ the total stellar luminosity of the Carina nebula (Smith 2006) and $d \sim 40 \mathrm{pc}\left(1^{\circ}\right)$ the distance of the dust grains from the sources. $<Q_{U V}>$ is the blackbody averaged absorption efficiency (assuming a blackbody temperature of $38 \times 10^{3} \mathrm{~K}$ ); the typical distance $d$ between the grains and the star has been estimated from the angular size of the nebula and its distance. This value, although obtained from a naive 
model, is in reasonable agreement with our results.

Equation (5) can be also used to estimate the mean distance of the dust grains from the center of the nebula once derived their temperatures. For a dust population of $34.5 \mathrm{~K}$ we find a distance of about $50 \mathrm{pc}$; in the presence of a colder dust population of about $18 \mathrm{~K}$ the distance would be about 200 pc.

Yonekura et al. (2005) estimate a mass of the molecular gas of about $3.5 \times 10^{5} \mathrm{M}_{\odot}$; since the molecular gas in the Carina nebula is about one third of the total mass budget (atomic + molecular gas + dust) following Smith \& Brooks (2007) we can estimate a total mass of about $10^{6} \mathrm{M}_{\odot}$; this compared with our estimated mass gives a gas-dust ratio of about 100, which is the canonical value assumed in this nebula (Morris et al. 1999; Smith \& Gehrz 2005; Smith \& Brooks 2007).

Our best estimate of the free-free emission, based mainly on the WMAP fluxes, is at $1.6 \sigma$ from the Parkes observation. One possibility to explain an excess flux observed in the Parkes data would be to suppose the presence of synchrotron radiation in the nebula, but this does not fit other long wavelength radio observations (Beard \& Kerr 1966; Jones 1973 ). More likely are effects related to the very different angular resolution of the Parkes data, 2.6 arcmin (Huchtmeier \& Day 1975), with respect to the WMAP observations. With a large beam the flux measured by WMAP in the pixels of the box might be overestimated, since every peripheral pixel includes the contribution of sources outside the integration box, but still inside the fraction of the beam spilling outside the box. The largest WMAP beam, $0^{\circ} .81$ FWHM, is still smaller than our integration box, so that the flux of the Carina Nebula lost in the sidelobes of the beam is negligible. However, if the $0^{\circ} .8$ beam is centered on the edge of the integration region, it might pick-up flux from sources outside the integration region, where half of the beam solid angle is lying. We have double checked this statement with numerical simulations. We have simulated a sky brightness distribution assuming that 
a single source, 30 arcmin in radius, is located in the center of our integration box. We have convolved this brightness with Gaussian beams with the same FWHM as the WMAP beams and integrated over our integration box to estimate the measured flux. The result is the same within less than $1 \%$ (at $94 \mathrm{GHz}$ ) (or up to $3 \%$ at $23 \mathrm{GHz}$ ) as in the case of an unsmoothed map, showing that the power lost in the sidelobes is negligible. Then we have added a second source, with 30 arcmin diameter and $20 \%$ of the maximum brightness of Carina, just outside our integration box. In this case we observe an overestimate of the measured fluxes which can be, again, from less than 1\% (at $94 \mathrm{GHz}$ ) to $3 \%$ (at $23 \mathrm{GHz}$ ) of the unsmoothed map. Therefore, the modest resolution of the low frequency channels of WMAP does not seem to be the reason of the deficit with respect to the Parkes data. Probably more important is the fact that the Parkes data do not cover our entire integration box (Smith \& Brooks 2007). From all this we conclude that a direct comparison is very difficult. This issue, however, does not affect the dust properties in the Carina nebula, which are the main focus of this paper.

The Carina nebula SED is well described by a four-parameter fit, although the lack of data between 240 and $730 \mu \mathrm{m}$ cannot exclude the presence of another dust component. Our 7-parameter fit gives an hint of this; nevertheless more data in the missing wavelength range are necessary to confirm it.

Our method of averaging over distinct background regions provides a background estimation generally in agreement with what was found by Smith \& Brooks (2007).

At wavelengths shorter than $60 \mu \mathrm{m}$, the emission in the nebula is dominated by polycyclic aromatic hydrocarbons (e.g., Rathborne et al. 2002) and warm dust grains at temperatures $\gtrsim 80 \mathrm{~K}$. At wavelengths longer than $60 \mu \mathrm{m}$, the flux from $\eta$ Carinae is negligible with respect to the integrated flux of the nebula. Cox et al. (1995) have shown that the composite IR SED of $\eta$ Carinae and the Homunculus Nebula is described by two 
modified Planck spectra $\nu^{\delta} B\left(\nu, T_{j}\right)$ with $\delta=1$ and $T_{j}=210,430 \mathrm{~K}$. At $20 \mu \mathrm{m}$, the peak of the flux density from the Homunculus nebula is about $10^{5} \mathrm{Jy}$, while about $10^{2} \mathrm{Jy}$ comes from the $35 \mathrm{~K}$ dust population of the more extended Carina Nebula (Smith \& Brooks 2007); at $60 \mu \mathrm{m}$ the emission of the Homunculus nebula drops to a value of about $2 \times 10^{4} \mathrm{Jy}$, while that of the $35 \mathrm{~K}$ dust increases to about $9 \times 10^{5} \mathrm{Jy}$. Among recent results (Smith et al. 2003), the ones based on Infrared Space Observatory observations (Morris et al. 1999) also confirm these findings. The global mass of the Carina nebula is not strongly affected by the mid-IR $(<60 \mu \mathrm{m})$ portion of the spectrum: the $200 \mathrm{~K}$ dust population adds a small contribution to the total mass budget which is negligible with respect to the large uncertain coming from colder (at 18 or $40 \mathrm{~K}$ ) dust grains populations.

Our hint for a second, colder, dust population with a temperature of about $18 \mathrm{~K}$ would be in good agreement with the analysis of the star-forming regions observed by PRONAOS, which predicts a high spectral index (about -2) for very cold regions (11-20) K (Dupac et al. 2001, 2003). The analysis of the millimetric continuous emission performed on the SIMBA-SCUBA star-forming regions shows a typical value of the spectral index equal to -2 , assuming dust temperatures higher than $10 \mathrm{~K}$ (Hill et al. 2006). However, the existence of a cold component cannot be excluded from the available data. It will be interesting to improve the SED of the Carina nebula with future observations, both better calibrated and at complementary wavelengths, either confirming or disproving hints for the existence of the very cold dust component, and its polarization properties.

The future balloon borne experiment PILOT (Bernard et al. 2007a,b) will observe the Carina nebula at the two wavelengths of 240 and $550 \mu \mathrm{m}$; observations at the first wavelength could confirm the flux measured by COBE-DIRBE, observations at the second wavelength will fill our missing range.

The ongoing Planck mission (Tauber et al. 2010) has measured the whole sky at 
nine wavelengths from 30 to $900 \mathrm{GHz}$, with unprecedented sensitivity, accurate $(<1 \%)$ calibration, and with angular resolution down to 5 arcmin; the SPIRE instrument on board of the Herschel satellite (Griffin et al. 2010) will also cover the region at 250, 350, and $500 \mu \mathrm{m}$. Once processed, these data will certainly solve the issue. The error in the background estimates, at wavelengths shorter than $2 \mathrm{~mm}$, are comparable to the difference between the SEDs of our one- and two-component best fits and larger than the Planck calibration errors; therefore they will not be the limiting factor for discriminating between the two models.

\section{Conclusion}

We have presented multi-wavelength observations of the Carina nebula. We compared our analysis to the Smith \& Brooks (2007) derivation of the SED. The SED of the nebula can be described as the sum of a graybody component, with a power-law emissivity, and an optically thin thermal bremsstrahlung. The parameters of the model are well constrained by the data: we find $e_{1}=\left(1.10_{-0.28}^{+0.38}\right) \times 10^{-3}, T_{1}=\left(34.5_{-1.8}^{+2.0}\right) \mathrm{K}, \alpha_{1}=-1.37_{-0.08}^{+0.09}$,

$a_{s}=1113_{-15}^{+12} \mathrm{Jy}$. Thanks to the B98 observations, in the range $730 \mu \mathrm{m}-3.3 \mathrm{~mm}$, we have been able to derive the spectral index $\alpha_{1}$ rather than assuming it equal to -1 . We also found, from our multi-component analysis, that the data are consistent with an inverse relation between spectral index and temperature. However, the improvement in the $\chi^{2}$ is not statistically significant. Our four-parameter results do not exclude the possibility that the FIR SED is described by two graybody components, the latter with a colder dust temperature and a steeper spectral index than the former. The small number of experimental data, compared to the number of free parameters does not allow a robust investigation of this hypothesis. This issue will be solved by the Planck/Herschel surveys. 


\section{Acknowledgements}

This activity has been supported by the Italian Space Agency projects BOOMERanG, OLIMPO, and LSPE, and by University of Rome La Sapienza. 


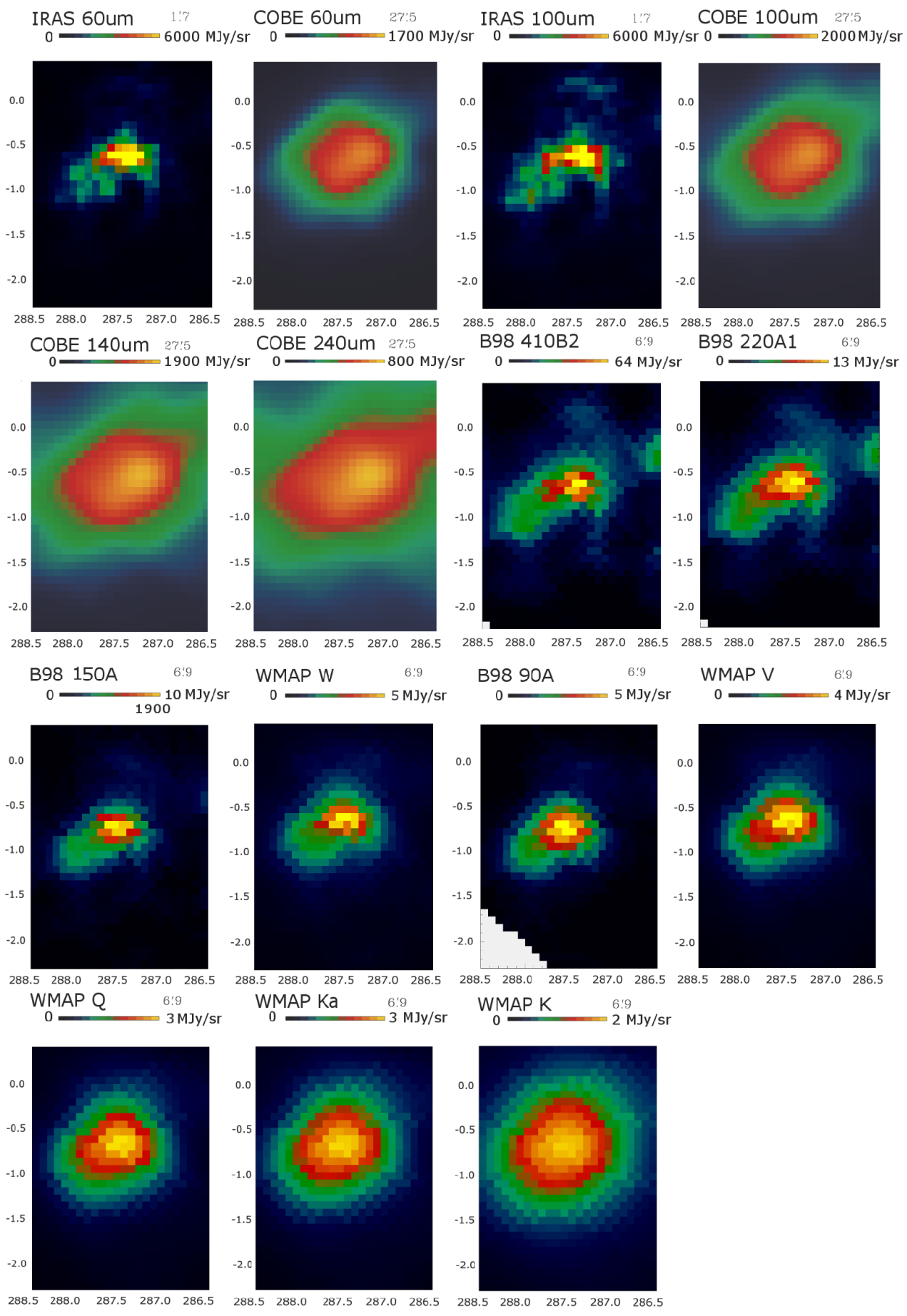

Fig. 1. - Wide-field images of the Carina nebula, from far-infrared (top left) to radio (bottom right) wavelengths. The ranges of the galactic coordinates (in degrees) reproduce the Smith \& Brooks (2007) integration box. 


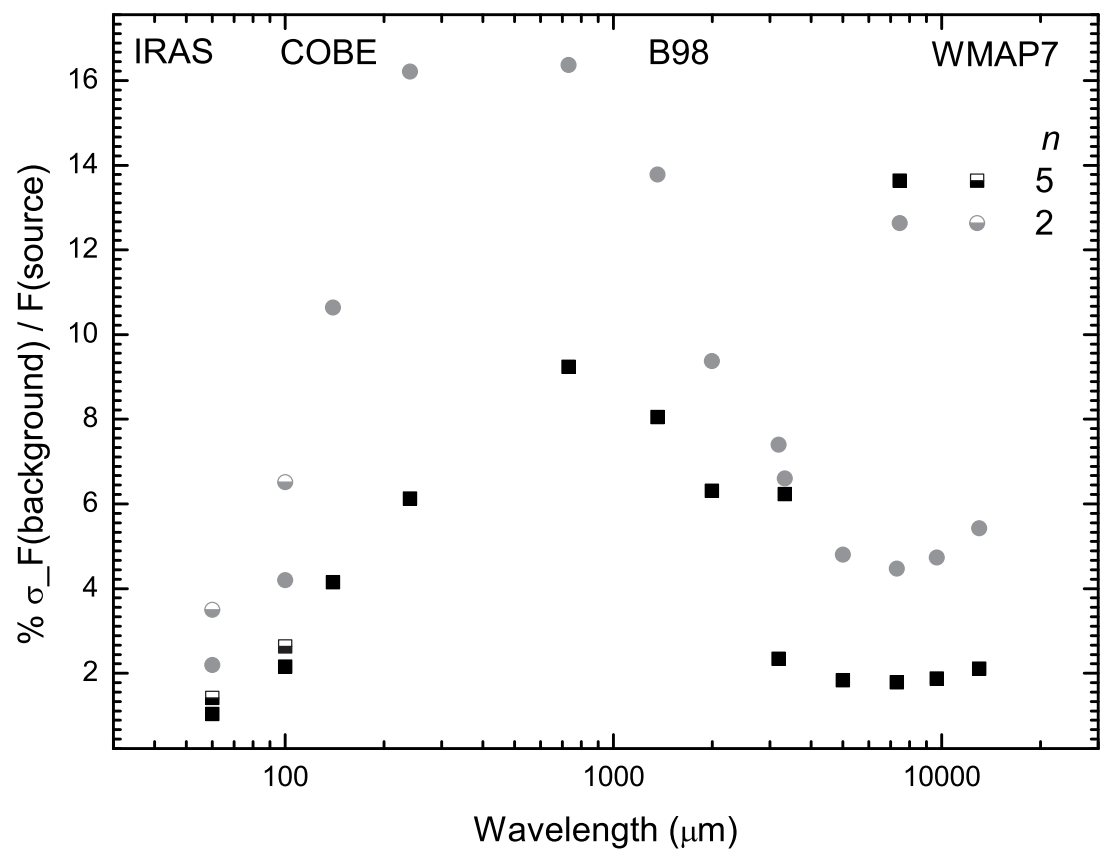

Fig. 2.- Standard deviation of the mean background contribution, $\sigma_{-} F$ (background), to the measured flux, F(source), corrected for the background, vs. the wavelength of the different data sets; the number $(n)$ labels the different number of regions considered for estimating the mean background. The IRAS points are labeled with half-colored symbols. 

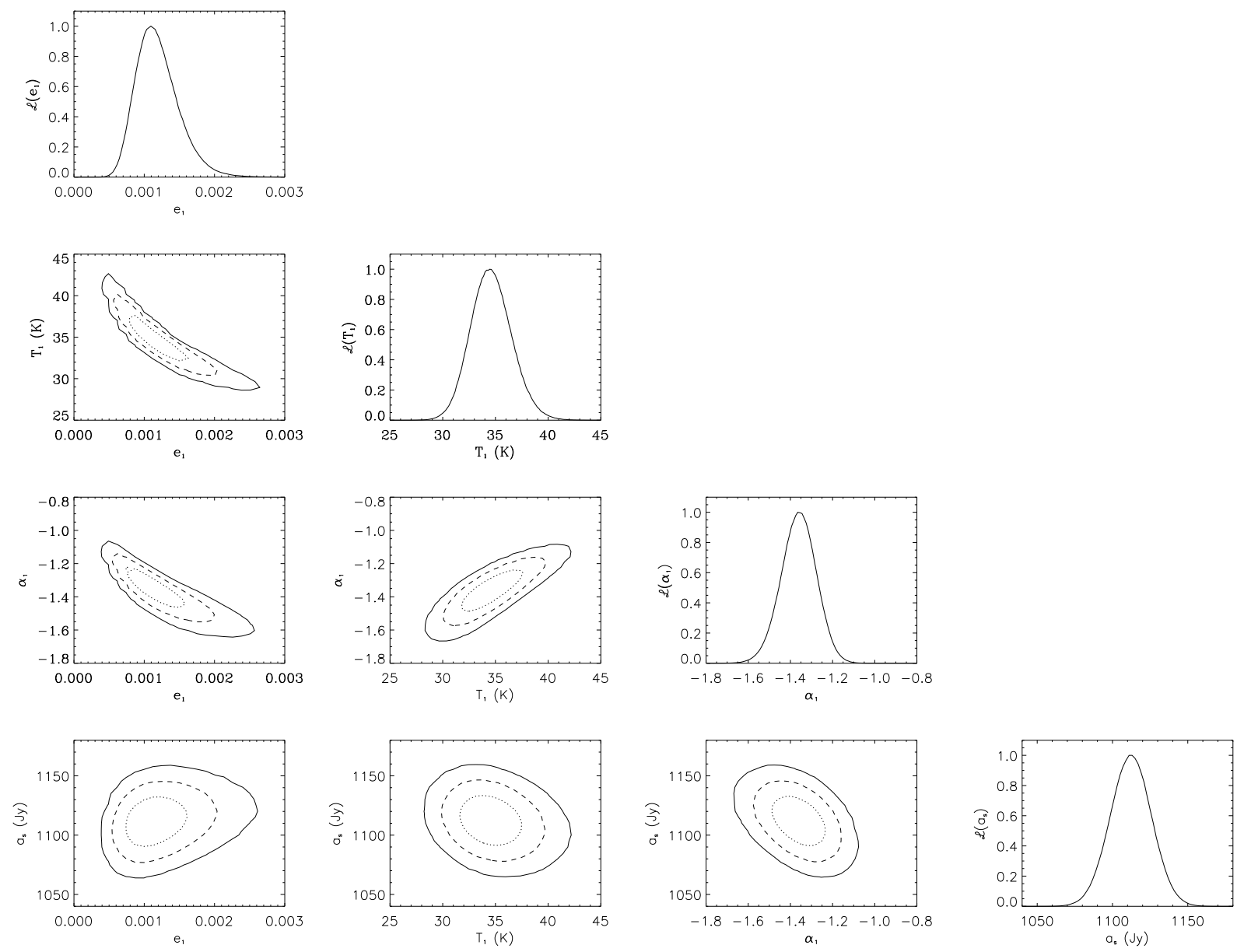

Fig. 3.- Contour plots of the joint probability density and likelihoods for the four parameters describing the SED of the Carina nebula. The dotted, dashed and solid regions represent confidence intervals for $68.3 \%, 95.4 \%$, and $99.7 \%$ CL, respectively. 


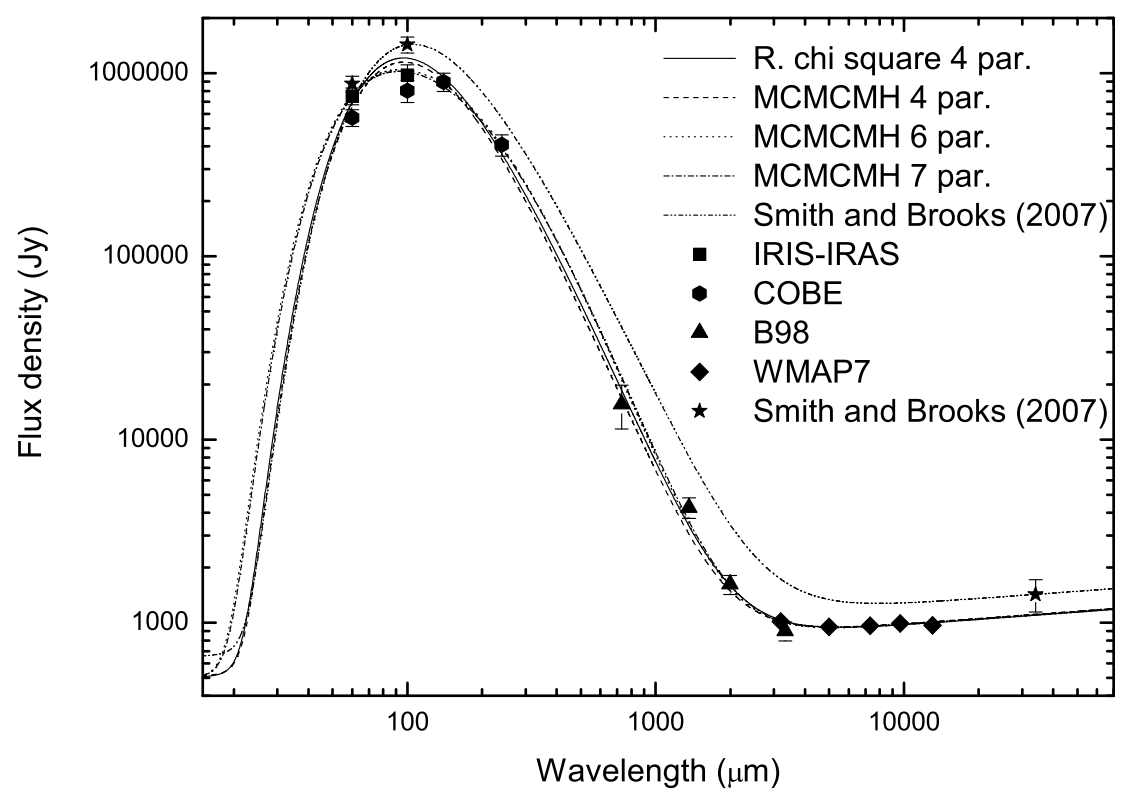

Fig. 4.- FIR to radio SED of the Carina nebula. In the legend we report, from top to bottom,: the best fit SED for four-parameter $\chi^{2}$ fit, the same for the MCMCMH with four, six, and seven parameters, and the Smith \& Brooks (2007) SED. The symbols represent the measured integrated fluxes. 


\section{REFERENCES}

Allen, D.A., \& Hillier, D.J. 1993, PASA, 10, 338

Andrieu, C., De Freitas, N., Doucet, A., et al. 2003, An Introduction to MCMC for Machine Learning, http://www.cs.princeton.edu/courses/archive/spring06/cos598C/papers /AndrieuFreitasDoucetJordan2003.pdf

Baumann, D., Jackson, M. G., Adshead, P., et al. 2009, in AIP Conf. Proc. 1141, CMB Polarization Workshop: Theory and Foregrounds, ed. S. Dodelson, D. Baumann, \& A. Cooray, et al. (Melville: AIP), 10

Beard, M., \& Kerr, F. J. 1966, AuJPh, 19, 875B

Beckwith, S. V. W., Sargent, A. I., Chini, R. S. and Gusten, R. 1990, AJ, 99, 924

Bennett, C. L., Bay, M., Halpern M., et al. 2003, ApJ, 538, 1

Bernard, J.-Ph., Ade, P. R. A., de Bernardis, P., et al. 2007a, in Sky Polarisation at Far-Infrared to RadioWavelengths: The Galactic Screen before the Cosmic Microwave Background, ed. F. Boulanger \& M.-A. Miville-Deschenes (Les Ulis: EDP Sciences), 189

Bernard, J.-Ph., Ade, P. R. A., de Bernardis, P., et al. 2007b, in Proc. 18th ESA Symp. on European Rocket and Balloon Programmes and Related Research, ed. A. Wilson (Noordwijk: ESA Communication Production Office), 499

Coble, K., Ade, P. A. R., Bock, J. J., et al. 2005, arXiv:astro-ph/0301599

Cox, P., Mezger, P. G., Sievers, A., et al. 1995, A\&A, 297, 168

Crill, B.P., et al. 2003, ApJS, 2, 527 
de Bernardis, P., Bucher, M., Burigana, C., Piccirillo, L., et al. 2009, Exper. Astron., 23, 5

Deschênes, M.-A. M. 2008, in SF2A-2008: Proc. AnnualMeeting of the French Society of Astronomy and Astrophysics, ed. C. Charbonnel, F. Combes, \& R. Samadi (Paris: Societe Francaise dAstronomie et dAstrophysique), 329

Deschênes, M.-A. M., \& Lagache G. 2005, ApJS, 157, 302

Draine, B. T. 1981, ApJ, 245, 880

Draine, B. T., \& Lee, H. M. 1984, ApJ, 285, 89

Dunkley, J., Amblard, A., Baccigalupi, C. et al. 2008, AIP Conf.Proc., 1141, CMB PolarizationWorkshop: Theory and Foregrounds (Melville, NY: AIP), 222

Dupac, X., Giard, M., Bernard, J.-P., et al. 2001, ApJ, 553, 604

Dupac, X., Bernard, J.P., Boudet, N., et al. 2003, A\&A, 404, L11

Fixsen, D.J., Cheng, E. S., Gales, J. M., et al. 1996, ApJ, 473, 576

Gorski, M.K., Hivon, E., Banday, A. J., et al. 2005, AJ, 622, 759

Griffin, M.J., Abergel, A., Abreu, A., et al. 2010, A\&A, 518, L3

Hauser, M.G., Kelsall, T., Leisawitz, D., \& Weiland, J. eds. 1998a, COBE Diffuse Infrared Background Experiment (DIRBE) Explanatory Supplement (Greenbelt: NASA/GSFC), http://lambda.gsfc.nasa.gov/product/cobe/dirbe_exsup.cfm

Hauser, M.G., Arendt, R. G., Kelsall, T., et al. 1998b, ApJ, 508, 25

Hildebrand, R. H., Dotson, J. L., Dowell, C. D., et al. 1999, ApJ, 516, 834

Hill, T., Thomson, M. A., Burton, M. G., et al. 2006, MNRAS, 368, 1223 
Hinshaw, G., Nolta, M. R., Bennett, C. L., et al. 2007, ApJS, 170, 288

Hinshaw, G., Weiland, J. L., Hill, R. S., et al. 2009, ApJS, 180, 225

Huchtmeier, W. K., \& Day, G. A. 1975, A\&A, 41, 153

Jones, B. B. 1973, AuJPh, 26545

Koike, C., Hasegawa, H., \& Manabe, A. 1980, Ap\&SS, 67, 495

Lazarian, A., \& Finkbeiner, D. 2003, New Astron. Rev., 47, 1107

Lazarian, A., \& Hoang, T. 2007, MNRAS, 378, 910

Lewis, A., \& Bridle, S. 2002, Phys.Rev.D, 66, 103511

MacKay, D.J.C. 2002, Information Theory, Inference, and Learning Algorithms (Cambridge: Cambridge Univ. Press), http://www.inference.phy.cam.ac.uk/mackay/itprnn/book.html

Martin, P. G. 2007, in Sky Polarisation at Far-Infrared toRadioWavelengths:The Galactic Screen before the CosmicMicrowave Background, ed. F. Boulanger \& M.-A. Miville-Deschenes (Les Ulis: EDP Sciences), 165

Masi, S., Ade, P. A. R., Bock, J. J., et al. 2001, ApJ, 553, L93

Mathis, J. S., \& Whiffen, G. 1989, ApJ, 341, 808

Montroy, T.,E., Ade, P. A. R., Bihary, R., et al. 2006, SPIE, 6267, 62670R

Morris, P.W., Waters, L. B. F. M., Barlow, M. J., et al. 1999, Nature, 402, 502

Neugebauer, G., et al. 1984, ApJ, 278, L1

Oxley , P., Ade, P. A. R., Baccigalupi, C., et al. 2004, in Proc. SPIE 2004, 5543, 320 
Paradis, D., Veneziani, M., Noriega-Crespo, A., et al. 2010, A\&A, 520, L8

Planck Collaboration: Ade, P. A., Aghanim, N, Arnaud, M. et al. 2011, arXiv:astro$\mathrm{ph} / 1101.2029$

Rathborne, J.M., Burton, M. G., Brooks, K. J., et al. 2002, MNRAS, 331, 85

Reichborn-Kjennerud, B., Aboobaker, A. M., Ade, P. A. R., et al. 2010, Proc. SPIE, 7741, $77411 \mathrm{C}$

Rieke, G. H., Young, E. T., Engelbracht, C. W., et al. 2004, ApJS, 154, 25

Ruhl, J. E., Ade, P. A. R., Bock, J. J., et al. 2003, ApJ, 599, 786

Smith, R.G 1987, MNRAS, 227, 943

Smith, N., Gehrz, R. D., Hinz, P. M., et al. 2003, AJ, 125, 1458

Smith, N., Bally, J., Brooks, K. J., et al. 2004, AJ, 127, 2793

Smith, N. 2005, ASP Conf. Ser.Ser. 332, The Fate of the Most Massive Stars, ed. R. Humphreys \& K. Stanek (San Francisco: ASP), 307

Smith, N., \& Gehrz, R. D. 2005, AJ, 129, 969

Smith, N. 2006, MNRAS, 367, 763

Smith, N., \& Brooks, K. J. 2007, MNRAS, 379, 1279

Smith, N., \& Brooks, K. J. 2008, Handbook of star forming region, Vol. 2, ed. B. Reipurth (Seattle, WA: ASP), 138

Tauber, J. A., et al. 2010, A\&A, 520, A1

Veneziani, M., et al. 2010, ApJ, 713, 959 
Yonekura, Y., Asayama, S., Kimura, K., et al. 2005, ApJ, 634, 476

Weintraub, D. A., Sandell, G., \& Duncan, W. D. 1989, ApJ, 340, L69

Wheelock, S., Gautier, T. N., Chillemi, J., et al. 1993, ISSA Explanatory Supplement, Technical Report (Pasadena: NASA/ESFC), http://irsa.ipac.caltech.edu/IRASdocs/issa.exp.sup/ 